Highlights of Astronomy, Vol. 12

International Astronomical Union, 2002

H. Rickman, ed.

\title{
Magnetic Fields in the Early Universe
}

\author{
Martin Rees \\ Institute of Astronomy, Madingley Rd, Cambridge CB3 OHA, UK
}

\begin{abstract}
It is argued that the seed field from the galactic dynamo comes from the first supernovae or stellar winds, or perhaps from early AGNs.
\end{abstract}

Cosmic magnetic fields probably owe their present pervasive strength to dynamo amplification. The requisite seed field seems to be generally 'taken for granted'. In many astrophysical contexts this confidence may be justifiable: if the dynamical (and amplification) timescale is short enough, there can be a huge number of $e$-foldings; a merely infinitesimal statistical fluctuation might then suffice. But the large-scale fields in disc galaxies seem to pose a less trivial problem. The amplification timescale may be $2 \times 10^{8}$ years; even by the present epoch there has been time for only $50 e$-foldings. The galactic field could not therefore have built up to its observed strength by the present day, unless the seed field strength were of order $10^{-25} \mathrm{G}$ - very weak, but not infinitesimal. Moreover, if it turned out that substantial fields existed even in high- $z$ galaxies whose discs may have only recently formed, the seed fields would need to have been correspondingly stronger.

The question of how quickly the interstellar magnetic field built up is germane to several aspects of galactic evolution. There is as much reason to believe that the absence of a magnetic field affects the stellar IMF as to believe that a lack of heavy elements does so (though the quantitative nature of the effect is as uncertain in the one case as the other). Also the behaviour of supernova remnants in high- $z$ galaxies is sensitive to the field strength (as is the trapping of cosmic rays, and the possibility of inflating loops into the halo - a process which may itself affect the efficiency of galactic dynamos).

It is generally agreed that battery mechanisms on galactic scales can only generate fields up to $10^{-19} \mathrm{G}$. I wish to suggest that a stronger seed field may originate from the first stars. I have presented this work more fully elsewhere; it relates also to earlier ideas by Zeldovich, Ruzmaikin, and Sokoloff, and to recent work by Kronberg and Lesch.

Even if a star initially had zero field, the Biermann battery could generate a seed field within it, on which dynamo amplification (by a huge number of factors of $e$ if necessary) could operate. If such a star expelled a strong wind or exploded as a supernova, then the ejecta could pervade several cubic parsecs with a field of strength of order $10^{-4} \mathrm{G}$. So the first few massive stars or supernovae could have created a weak field, perhaps at a redshift of order 10. These fields would therefore already be present in the material that formed galactic discs their early diffusion would be closely linked to the diffusion of the first heavy elements. Provided that the large-scale modes could be preferentially amplified, 
these stellar-generated fields would be adequate seeds for a galactic dynamo. For a quantitative estimate, note that each hemisphere of the Crab Nebula contains an (equal and opposite) flux of order $10^{34} \mathrm{G} \mathrm{cm}^{2}$. If $N$ similar remnants formed, in for instance, a young galactic disc, the net flux would then be larger by some power $x$ of $N$. The appropriate value for $x$ is not obvious. The net effect depends on the two hemispheres evolving differently - otherwise the net flux cancels out. To assume that $x=1 / 2$ may therefore be over optimistic. A better guess might be $x=1 / 3$. This is appropriate if the remnants are randomly oriented, and the galaxy can be modelled as the interior of a surface which slices a fraction $N$ of the remnants. As an example, if $N=10^{6}$, the large-scale component of the field in a protogalactic disc of $10 \mathrm{kpc}$ radius would be $3 \times 10^{-8}-3 \times 10^{-9} \mathrm{G}$, for $x$ in the range $1 / 3-1 / 2$.

A separate possibility is that the fields come from radio lobes. Some high- $z$ radio sources contain ordered fields of $10^{-5} \mathrm{G}$ on scales of tens of kiloparsecs, implying a flux of order $10^{42} \mathrm{G} \mathrm{cm}^{2}$. Some such sources may well have formed exceptionally early, when the formation of typical galaxies (especially those with discs) still lay in the future. The fields in the lobes of radio galaxies could have been generated in the active nucleus of the associated galaxy and expelled along collimated jets (resembling a scaled-up and directional version of the relativistic pulsar wind that generates the field of the Crab Nebula). In the nucleus itself, the amplification timescale may be shorter than a year.

If the infalling material that makes a galactic disc had been 'contaminated' by a fraction $f$ of a radio source lobe, the large-scale component of the seed field would be $3 \times 10^{-8}\left(f / 10^{-4}\right)$ G. So only a small value of $f$ might suffice.

I have focussed on the question of the seed field for the galactic dynamo: this is a more challenging question than the seeding of smaller-scale cosmic dynamos because the galactic dynamo timescale is so long, and the amplification correspondingly slow. (And I have assumed, of course, that the galactic dynamo mechanism is indeed efficient - the problem is obviously far worse if it is not.) Because of the importance of a magnetic field in star formation, we have little chance of really understanding what a high-redshift galaxy should look like until these issues have been given a good deal more attention by experts in cosmic magnetism. 